\title{
Behavioural discrimination of noxious stimuli in infants is dependent on brain maturation
}

Gabrielle Green $^{\mathrm{a}}$, Caroline Hartley ${ }^{\mathrm{a}}$, Amy Hoskin ${ }^{\mathrm{a}}$, Eugene Duff ${ }^{\mathrm{a}}$, Adam Shriver ${ }^{\mathrm{b}}$, Dominic Wilkinson ${ }^{\mathrm{b}}$, Eleri Adams ${ }^{\mathrm{a}}$, Richard Rogers ${ }^{c}$, Fiona Moultrie ${ }^{a}$, Rebeccah Slater ${ }^{a, \star}$

\begin{abstract}
Changes in facial expression are an essential form of social communication and in nonverbal infants are often used to alert care providers to pain-related distress. However, studies of early human brain development suggest that premature infants aged less than 34 weeks' gestation do not display discriminative brain activity patterns to equally salient noxious and innocuous events. Here we examine the development of facial expression in 105 infants, aged between 28 and 42 weeks' gestation. We show that the presence of facial expression change after noxious and innocuous stimulation is age-dependent and that discriminative facial expressions emerge from approximately 33 weeks' gestation. In a subset of 49 infants, we also recorded EEG brain activity and demonstrated that the temporal emergence of facial discrimination mirrors the developmental profile of the brain's ability to generate discriminative responses. Furthermore, within individual infants, the ability to display discriminative facial expressions is significantly related to brain response maturity. These data demonstrate that the emergence of behavioural discrimination in early human life corresponds to our brain's ability to discriminate noxious and innocuous events and raises fundamental questions as to how best to interpret infant behaviours when measuring and treating pain in premature infants.
\end{abstract}

Keywords: Facial expression, Behaviour, EEG, Preterm, Noxious, Innocuous, Evoked response, Brain activity

\section{Introduction}

Facial expressions in infants facilitate social interaction ${ }^{16}$ and provide a mechanism by which infants can alert care providers to their pain or distress. ${ }^{33}$ This immature form of social communication elicits intervention and ultimately protects infants from aversive situations. Hospitalised infants regularly undergo painful medical procedures, ${ }^{7}$ and facial expressions form the cornerstone of infant pain assessment. ${ }^{28}$ Pain perception and observed pain-related facial expressions have been related in adults. ${ }^{27}$ However, this may not be the case in premature infants due to the immaturity of the developing nervous system. ${ }^{11,19}$ In the most premature infants, facial expressions can be observed after procedural touch stimulation, ${ }^{15}$ and it is unclear whether premature infants display discriminative behaviours after

Sponsorships or competing interests that may be relevant to content are disclosed at the end of this article.

G. Green and C. Hartley have contributed equally.

a Department of Paediatrics, University of Oxford, Oxford, United Kingdom, ${ }^{b}$ The Oxford Uehiro Centre for Practical Ethics, University of Oxford, Oxford, United Kingdom, ${ }^{c}$ Nuffield Department of Anaesthesia, John Radcliffe Hospital, Oxford, United Kingdom

*Corresponding author. Address: Department of Paediatrics, Children's Hospital, John Radcliffe, Oxford OX3 9DU, United Kingdom. Tel.: 01865234229. E-mail address: rebeccah.slater@paediarics.ox.ac.uk (R. Slater).

Supplemental digital content is available for this article. Direct URL citations appear in the printed text and are provided in the HTML and PDF versions of this article on the journal's Web site (www. painjournalonline.com).

PAIN 160 (2019) 493-500

Copyright @ 2018 The Author(s). Published by Wolters Kluwer Health, Inc. on behalf of the International Association for the Study of Pain. This is an open access article distributed under the Creative Commons Attribution License 4.0 (CCBY), which permits unrestricted use, distribution, and reproduction in any medium, provided the original work is properly cited.

http://dx.doi.org/10.1097/j.pain.0000000000001425 salient noxious and innocuous events. ${ }^{1}$ To improve the measurement of pain in nonverbal hospitalised infants, we need a greater understanding of how pain-related facial expressions emerge and develop in early life.

Discriminative patterns of evoked brain activity emerge across the preterm period. In younger gestation infants, nondiscriminative activity known as a delta brush, which may have origins in the insula, ${ }^{3}$ is evoked by both innocuous and noxious inputs, ${ }^{12}$ as well as auditory and visual stimuli. ${ }^{9,10}$ By contrast, older infants from approximately 34 to 35 weeks' gestation generate noxiousevoked brain activity with specific morphology that is not evoked by visual, tactile, or auditory inputs. ${ }^{12,18} \mathrm{We}$ hypothesised that, consistent with the maturation of discriminative patterns of brain activity, changes in facial expression discriminating between noxious and innocuous stimulation will emerge during the preterm period. We examined the development of facial expressions in infants aged between 28 and 41 weeks' gestation, investigating their responses to acute procedural noxious and innocuous stimulation. In a subset of infants, we recorded their brain activity responses using EEG to determine how facial expression relates to the maturation of evoked brain activity.

\section{Methods}

\subsection{Participants}

Between April 2012 and May 2017, a total of 122 infants were recruited from the Newborn Care Unit and Maternity wards of the John Radcliffe Hospital, Oxford University Hospital NHS Foundation Trust, Oxford, United Kingdom. Infants were born between 23 and 42 weeks' gestation and were between 28 and 42 weeks' gestational age at the time of study. One hundred and five infants were included in an analysis of facial expressions and 49 infants were included in an analysis of evoked brain activity across the preterm 
period. A sample of 18 term infants were used to characterise the non-modality-specific pattern of EEG activity recorded in response to both noxious and tactile stimulation, and to derive an EEG template of this sensory-evoked activity (see supplementary Figure 1 for further detail, available at http://links.Iww.com/PAIN/A686).

Infants were not included in the study if they had documented neurological malformations, intraventricular hemorrage (IVH) greater than grade 2, or a history of maternal substance abuse. At the time of study, all infants were clinically stable and not requiring invasive ventilation. None of the infants had received analgesics or sedatives in the preceding 72 hours. Infant demographics were recorded at the time of study from the clinical notes and are described in Table 1. To estimate cumulative pain exposure, we retrospectively reviewed the number of oropharyngeal aspirations, tracheal aspirations, and tissue-damaging procedures performed for blood taking (which included heel lances, venipuncture, and intravenous cannulation) that were documented in the electronic and paper clinical records between birth and the time of study. These procedures were selected as they are among the top 6 most common painful procedures experienced by infants in neonatal intensive care. ${ }^{7}$ They have been used in previous studies ${ }^{19}$ and are clinically well documented, facilitating retrospective review. We did not ascertain the number of attempts for each procedure (as this information is not available retrospectively); however, the estimate produced by this method provides a consistent way of comparing infant pain exposure across the study population.

Ethical approval was obtained from the National Research Ethics Service (references: 12/SC/0447 and 11/LO/0350) and written informed parental consent was gained before each participant was studied. The study was conducted in accordance

\section{Table 1}

Infant demographics.

\begin{tabular}{ll}
\hline Total infants & 122 \\
\hline Gestational age at birth (wk) & 31.9 (range: 23.0-42.0) \\
\hline Gestational age at time of study (wk) & 36.5 (range: 28.0-42.7) \\
\hline Postnatal age at time of study (d) & 9 (3-26) \\
\hline Birth weight (g) & 1830 (990-3340) \\
\hline Weight at study (g) & 2053 (1464-3380) \\
\hline Male infants (\%) & $69(57)$ \\
\hline Multiple gestation infants (\%) & $37(30)$ \\
\hline Spontaneous vaginal deliveries (\%) & $48(39)$ \\
\hline Assisted/caesarean deliveries (\%) & $74(61)$ \\
\hline Apgar score at 5 minutes & $10(8-10)$ \\
\hline Infants admitted to NICU (\%) & $80(66)$ \\
\hline Infants ventilated during admission (\%) & $39(32)$ \\
\hline Days of ventilation & $0(0-1)$ \\
\hline Infants who had previously received morphine & $26(21)$ \\
\hline during admission (\%) & $10(8)$ \\
\hline Infants with grade 1 or 2 IVH (\%) & $3(2)$ \\
\hline Infants with a history of previous surgery (\%) & $38(31)$ \\
\hline Infants with a previous diagnosis of postnatal \\
infection (\%)
\end{tabular}

with the standards set by the Declaration of Helsinki and Good Clinical Practice guidelines.

\subsection{Experimental procedures}

\subsubsection{Heel lancing and control heel lance}

All heel lances were performed when clinically required as part of the infant's medical care. Heel lances were not performed solely for the purpose of the study. Care was taken to ensure that the infants were given appropriate comfort, such as swaddling or containment holding, according to gestational age and parental preference. When the infants were swaddled, they were laid on a cotton cloth with their arms crossed over their chest in a relaxed position. The ends of the cloth were then crossed over the infant's body and arms, and tucked beneath the opposing side. The swaddling cloth restricted gross body movements and held the infant securely and comfortingly in a flexed position, without covering their feet to allow access for blood taking. The choice of foot for heel lancing was based on clinical judgment and not controlled during the experiment. Heel lances were performed on the medial or lateral plantar surface of the heel. In term infants, a BD Microtainer Quikheel Infant Lancet (Becton, Dickinson and Company, Franklin Lakes, NJ) with a penetration depth of $1.0 \mathrm{~mm}$ was used and, in preterm infants, a BD Quikheel Preemie Lancet with a penetration depth of $0.85 \mathrm{~mm}$ was used. After the lance, the foot was not squeezed for 30 seconds to ensure the observed response was only to the stimulus applied. Before the lance, a control lance was performed whereby the lancet was rotated by $90^{\circ}$ and held against the infant's foot so that when the lance was released, there was no contact with the infant's heel. A video camera was used to record facial expressions throughout the procedure for post hoc analysis. The timings of the lance and control lance were marked on the video using an LED, which flashed when the person performing the lance pressed a foot pedal at the point of stimulation.

\subsubsection{EEG recordings}

In 49 infants, brain activity was recorded during the stimuli using EEG. EEG from DC to $400 \mathrm{~Hz}$ was acquired with a SynAmps RT 64-channel EEG/EP system (Compumedics Neuroscan). Activity was recorded with a sampling rate of $2000 \mathrm{~Hz}$ using CURRYscan7 neuroimaging suite (Compumedics Neuroscan). To optimise contact with the scalp, the skin was gently rubbed with EEG preparation gel (NuPrep gel; D.O. Weaver and Co, Aurora, $\mathrm{CO})$ before electrode placement and application of EEG conductive paste (Elefix EEG paste; Nihon Kohden). EEG was recorded at the $\mathrm{Cz}, \mathrm{CPz}, \mathrm{C} 3, \mathrm{C} 4, \mathrm{FCz}, \mathrm{Oz}$, T3, and T4 electrode sites, with the reference electrode at $\mathrm{Fz}$ and a ground electrode on the forehead. In 11 infants, EEG was recorded at Cz, CPz, C3, and C4 only. Stimuli were time locked to EEG recordings using an accelerometer as previously described. ${ }^{46}$

\subsection{Analysis}

\subsubsection{Facial expression scores}

Analysis of the facial expression was undertaken after acquisition by research assistants, who had been trained in Premature Infant Pain Profile-Revised (PIPP-R) scoring and who had achieved high levels of interrater and intrarater reliability before the study analysis. The baseline behavioural state was scored in the 15 seconds preceding the control lance and again in the 15 seconds preceding the heel lance. A score between 0 and 3 was 
given according to whether the infant was active and awake, quiet and awake, active and asleep, or quiet and asleep, respectively, as per the Premature Infant Pain Profile-Revised (PIPP-R). ${ }^{42}$

The presence of 3 facial expressions (brow bulge, eye squeeze, and nasolabial furrow) was assessed in the 30 seconds after both the control lance and heel lance. The duration of facial expression activity was defined as the duration during which any of the 3 facial expressions were observed, whether together or individually, to a maximum of 30 seconds. The duration of facial expressions was timed using a stopwatch. If the infant stopped displaying a facial expression, the timer was paused, and if any expression was seen again during the 30-second period, the timer was restarted to give a cumulative time. An infant was considered to have a facial expression response to a stimulus if the infant displayed any response during the 30-second period (ie, if the duration of the response was greater than 0 ).

Each facial expression was also taken individually to calculate a facial expression score using the facial component of the PIPP/ PIPP-R. ${ }^{41,42}$ A score between 0 and 3 for each facial expression was given accordingly, giving a total score between 0 and 9 . Observers were blinded to the stimulus type when scoring the videos. Interrater and intrarater reliability were calculated using intraclass correlation of the PIPP scores. This was performed for $20 \%$ of the videos that were rescored by the first observer, and $35 \%$ of the videos that were rescored by a second independent observer. Videos were selected at random for rescoring. The intrarater reliability was 0.95 and the interrater reliability was 0.96 .

\subsubsection{EEG analysis}

EEG activity was filtered from 0.5 to $70 \mathrm{~Hz}$ with a notch filter at 50 $\mathrm{Hz}$. The data were epoched from 4 seconds before and after the stimulus, and baseline corrected to the prestimulus mean. Individual EEG channels contaminated with artefacts, such as movement artefact, were removed from the analysis.

Noxious-specific brain activity was identified from recordings at the $\mathrm{Cz}$ electrode site using a previously described template of noxious-evoked brain activity. ${ }^{18}$ Individual EEG traces were first Woody filtered by a maximum jitter of $\pm 50 \mathrm{~ms}$ to achieve maximal correlation of the data and the template. The template was then projected onto the data in the time window from 400 to $700 \mathrm{~ms}$ after the stimulus to obtain the magnitude of the noxious-evoked brain activity, as previously described. ${ }^{18}$ The same process was repeated with background EEG recordings-where the infant's foot was gently held but no stimuli were applied. This gave a distribution of the magnitude of the brain activity within the background data, and a threshold of $80 \%$ of this background distribution was set as the threshold for noxious-specific brain activity (a value of 0.34). Thus, if the response to the heel lance was above this threshold in any given infant, noxious-specific brain activity was said to have occurred.

In term infants, both tactile and noxious stimuli have been shown to evoke an earlier potential approximately $250 \mathrm{~ms}$ after the stimulus. ${ }^{12,38}$ An independent sample of 18 term infants (which were the same group of infants used to derive the template of noxious-evoked brain activity in a previous publication ${ }^{18}$ ) was used to characterise this response at the $\mathrm{Cz}$ electrode. Activity in the background period and in response to a control lance, heel lance, experimental noxious stimulation (128 mN PinPrick; MRC systems), and experimental tactile stimulation (modified tendon hammer) was epoched from 1 second before to 1.5 seconds after the stimulus, filtered from 0.5 to $8 \mathrm{~Hz}$ to allow the response to be characterised without being affected by artefacts, and baseline corrected to the prestimulus mean (for further details of the stimuli and experimental design, see Study 1 from Hartley et al. ${ }^{18}$ ). The data were first Woody filtered to the average response in the time window 100 to $300 \mathrm{~ms}$ after the stimulus, with a maximum jitter of $\pm 50 \mathrm{~ms}$ to account for variation in latency with individual infants. Principal Component Analysis was then conducted on the data in the same time window. The first 2 principal components accounted for $95 \%$ of the variance and were therefore the only components considered. The weights of the first component were significantly higher in response to the tactile and noxious stimuli compared with background activity, indicating that the component was related to the stimulation $(P<0.05$, linear mixedeffects analysis with Tukey post hoc comparisons). By contrast, the weights of the second principal component were not significantly different between modalities $(P=0.23$, linear mixed-effects model); so, the first principal component was selected as the template of the sensory-evoked potential. This template was then projected onto individual trials in the study data set at the $\mathrm{Cz}$ electrode using singular value decomposition to calculate the magnitude of the sensory-evoked potential within each individual trial (data were first Woody filtered to the template in the time window $100-300$ ms after the stimulus). ${ }^{12,18}$ Similarly, to the noxious-specific brain activity, this process was also performed with the background brain activity to obtain a distribution of the background data. A threshold of $80 \%$ of this background distribution (a value of 0.50 ) was used to define the occurrence of the sensory-evoked potential within individual trials.

The presence of delta brushes was investigated using a previously described burst detection method based on the cooccurrence of slow waves $(0.5-2 \mathrm{~Hz})$ and higher frequency activity $(8-22 \mathrm{~Hz}) .{ }^{17}$ Delta brushes were said to occur in response to stimulation if they occurred at any electrode site and the start of the nested higher-frequency activity was within 2 seconds of the point of stimulation (this point was taken because the algorithm detects a delta brush as the co-occurrence of the slow- and highfrequency activity and so defines the start as the start of the highfrequency activity, not the slow-frequency activity that will begin earlier). ${ }^{12}$

\subsection{Statistical analysis}

The MATLAB programming environment was used to conduct all statistical analyses. Generalised linear regressions with logit link functions were used to describe the proportion of infants with facial expression responses and different patterns of brain activity, across gestational age. For this, proportions were first calculated in 2-week intervals (starting at 28-30 weeks) with intervals overlapping by 1 week. To ensure that the number of infants was well distributed across the age range, a minimum of 12 infants were included in each 2-week interval. A generalised linear regression was also used to assess the proportion of infants with discriminative facial expressions according to brain response maturity. Comparison of the duration of facial expression response to the innocuous and noxious stimulation was performed using Wilcoxon signed rank tests.

Gaussian process modelling was used to model the development of response probabilities to innocuous and noxious stimuli with age, to assess the point of divergence of facial expression discrimination. ${ }^{35}$ For each of the 2 stimuli independently, the probability of infants responding to the stimulus was modelled across age using a squared exponential with automatic relevance determination covariance function, with a characteristic length scale of 3.5 weeks and an SD of 1 . The Gaussian process 
models smooth changes of the likelihood of the infants to respond to the stimulus with age. An error function likelihood was used, and inference was achieved using expectation propagation.

Once the models were fitted, the distribution of modelled response likelihoods at any given age were compared and used to assess the divergence of response probabilities for the 2 stimuli. Using a Gaussian approximation to the standard error of response likelihoods, approximate $P$-values were generated to quantify the magnitude of this difference at a particular age. Note that these $P$-values do not reflect independent statistical tests, but indicate, given the model, the expected proportion of experiments where the difference in response likelihoods across stimuli matches that observed.

\section{Results}

We recorded the facial expressions of 105 clinically stable infants, aged between 28 and 41 weeks' gestation, during a clinically required noxious heel lance (for blood testing) and an innocuous control procedure (control heel lance). The presence and duration of 3 facial expressions-brow bulge, nasolabial furrow, and eye squeeze, as described in the Premature Infant Pain Profile ${ }^{41,42}$ were assessed in the 30 seconds after the stimuli. Infant demographics are shown in Table 1.

Overall, $24 \%$ of infants displayed a facial expression response after the innocuous stimulus and 69\% displayed a facial expression response to noxious stimulation. However, the likelihood that facial responses were observed was agedependent (Fig. 1A). The proportion of infants who displayed a noxious-evoked facial expression significantly increased with gestational age $(P=0.0005$, generalised linear regression, coefficient $\beta=0.16,95 \%$ confidence interval [Cl]: 0.07-0.24, Fig. 1A), whereas the proportion of infants who displayed a facial expression to the innocuous stimulation significantly decreased with gestational age $(P=0.014, \beta=-0.11,95 \% \mathrm{Cl}$ : -0.21 to -0.02 , Fig. $1 \mathrm{~A}$ ).
Using Gaussian process modelling, ${ }^{35}$ a supervised machine learning method that can model changes in the probabilistic state of populations, we identified that the responses diverge from 33.0 weeks' gestation, at which point there is a 95\% probability that the average proportion of infants responding to noxious stimuli is greater than the average proportion of infants responding to the innocuous stimuli (Fig. 1A). By 33.9 weeks' gestation, this probability increases to $99 \%$. Before 32 weeks' gestation, there is a substantial overlap in the distributions of infants responding to the noxious and innocuous stimuli $(P>0.1)$.

The duration of the facial expressions also demonstrated a similar developmental profile. In infants younger than 32 weeks' gestation, there was no significant difference in the duration of the evoked facial expression after either stimuli (median difference: 2.35, 95\% Cl: $-1.10-8.00, P=0.16, N=26$, Wilcoxon signed rank test, Fig. 1B). By contrast, in infants older than 33.9 weeks' gestation, the duration of the noxious-evoked facial activity was significantly greater than the duration of facial activity evoked by the innocuous stimulation (median difference: $7.85,95 \% \mathrm{Cl}$ : 5.75-11.10, $P<0.001, N=65$, Wilcoxon signed rank test, Fig. 1B). This suggests that age-dependent maturation of discriminative facial expressions may permit older infants to better communicate their pain experience.

The age of emergence of the development of discriminative modality-specific brain activity patterns has been relatively well characterised $^{12,19}$ and seems consistent with the behavioural maturation observed here. We recorded brain activity responses to the noxious and innocuous stimuli in a subset of 49 infants using EEG, and assessed the presence of (1) noxious-specific brain activity, (2) sensory-evoked brain activity (an evoked potential elicited by either noxious or innocuous stimulation), ${ }^{38}$ and (3) delta brushes (see Methods). Consistent with previous observations in premature infants, ${ }^{9,10,12}$ we observed that younger, more premature, infants are more likely to display delta brushes and that older infants more likely display noxious-specific activity after the heel lance. Overall, $67 \%$ of infants in this population exhibited noxious-specific brain activity after the heel
A

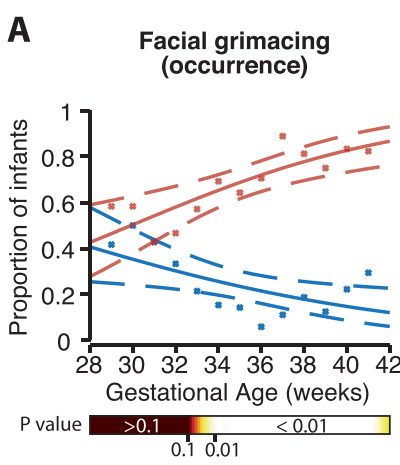

B

- Noxious stimulation

Innocuous stimulation

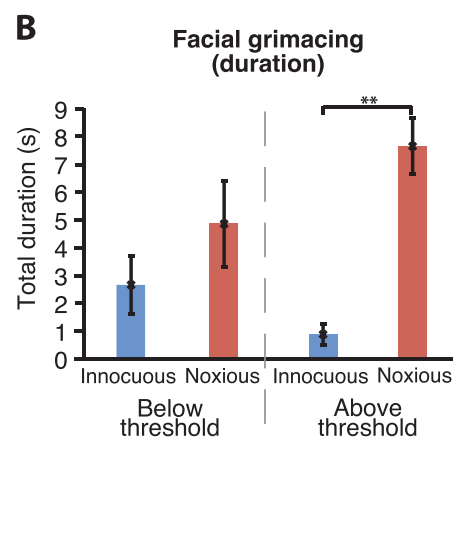

C

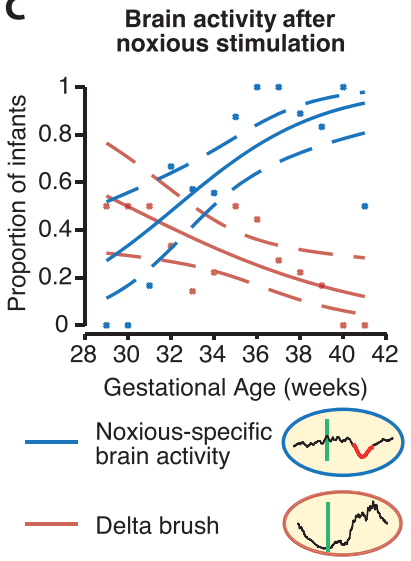

Figure 1. Facial expression and brain activity discrimination emerges with increasing gestational age. (A) The proportion of infants who displayed facial expressions after noxious stimulation (red) and innocuous stimulation (blue) according to gestational age. Proportions were calculated in 2-week intervals, with intervals overlapping by 1 week. The fit from a generalised linear model (solid lines) and $90 \%$ confidence intervals (dashed lines) are overlaid. The bottom colour bar indicates the $P$-values for the difference in proportions between the 2 groups calculated using Gaussian process modelling. The proportion of infants younger than 32 weeks' gestation who responded to the noxious stimuli shows substantial overlap with the proportion who responded to innocuous stimulation $(P>0.1)$, whereas in infants older than 33.9 weeks' gestation, infants are significantly more likely to display facial expressions after noxious stimulation compared with innocuous stimulation ( $P<0.01$, Gaussian process model). (B) Infants younger than 32 weeks also had no significant difference in the duration of their facial expression after noxious or innocuous stimulation, whereas facial expression responses of infants older than 33.9 weeks' gestation were significantly longer in duration after the noxious stimulation compared with the innocuous stimulation ( ${ }^{\star *} P<0.001$ ). (C) The proportion of infants who exhibited noxious-specific brain activity (blue) or non-modality-specific delta brush responses (red) to the noxious stimulation. (D) The proportion of infants who exhibited a sensory-evoked potential (black) and delta brushes (red) after innocuous stimulation. 
lance, and the proportion significantly increased with gestational age $(P=0.0005$, generalised linear regression, $\beta=0.30,95 \% \mathrm{Cl}$ : $0.14-0.48$, Fig. 1C). Only 1 of the 8 infants (12.5\%) who were younger than 32 weeks' gestation had noxious-specific brain activity after the heel lance, compared with 27 of 33 (82\%) infants older than 33.9 weeks' gestation. In a recent article, we did not report any noxious-specific brain activity in infants younger than 32 weeks' gestation, ${ }^{19}$ but this is likely to reflect the small sample size, and the results here demonstrate that although it is possible to observe noxious-specific brain activity at this age, consistent with previous reports, ${ }^{12}$ this occurs with increasingly lower frequency in younger gestational infants. ${ }^{44}$ In the most premature infants, delta brush activity was more likely to be evoked in response to both the noxious and innocuous stimuli, rather than the more mature modality-specific activity patterns (Figs. 1C, D). These data confirm that the emergence of modality-specific brain activity and discriminative facial expressions occur over the same developmental time window, at approximately 33 weeks' gestation.

In the subset of 46 infants who had both facial expression and brain activity recorded, 44 infants had facial expression and EEG activity recorded in response to both the control heel lance and the heel lance without artefact. We investigated whether the infants' ability to display behavioural discrimination was dependent on the maturity of their brain activity responses. We used a decision tree to categorise infants based on the maturity of their brain activity (Fig. 2A). We defined infants with the least mature brain responses as those with only evoked delta brushes, and infants with the most mature responses as those with both noxious-specific brain activity in response to the heel lance and a sensory-evoked potential in response to either the noxious or innocuous stimulation. Two infants who did not display any type of evoked response, and therefore whose response maturity could not be classified, were excluded from the analysis, leaving 42 infants in the decision tree. The proportion of infants who displayed discriminative facial expressions (defined as a behavioural response to the noxious but not the innocuous stimulus) in each group was calculated.

The infants' ability to display discriminative facial expressions significantly increased with increasing maturity of the infants' brain responses $(P=0.016$, generalised linear regression, $\beta=$ 0.49, 95\% Cl: 0.11-0.93, Fig. 2B). Seventeen of the 23 infants (74\%) with mature brain activity patterns (noxious-specific and sensory-evoked brain activity) displayed discriminative facial expressions. By contrast, in infants with immature brain activity (ie, where noxious-specific brain activity or sensory-evoked brain activity were not generated), only 7 of the 19 infants (37\%) displayed discriminative facial expression responses. This demonstrates that an infant's ability to display discriminative facial expressions is related to their brain maturity.

\section{Discussion}

In this study, we demonstrate that discriminative facial expressions emerge at approximately 33 weeks' gestation. Older infants are more likely to display facial expressions only to the noxious stimuli, whereas younger infants are as likely to display facial expressions to either stimulation with no difference in the response duration. Overall, we demonstrate that the emergence of discriminative facial expressions coincides with the maturation of brain activity responses.

Discriminative facial expressions to tactile and noxious stimulation have previously been reported in premature infants from 26 to 31 weeks' gestation, ${ }^{24}$ which contrasts with the results reported here. However, this is likely due to the differences in experimental design. Although the innocuous tactile stimulus in our study was elicited by rotating the lancet by $90^{\circ}$ and releasing the blade, Johnston et al. compared noxious heel lancing with nurse touch. The innocuous stimulus we used did not pierce the infant's skin, but was not comparable to nurse touch as it replicated all other salient aspects of the clinical heel lance procedure. Therefore, there is no suggestion from this study that positive tactile stimulation should be discouraged in the care of premature infants. Until recently, repeated tactile stimulation in extremely preterm infants was thought to be associated with hypoxemia, ${ }^{29}$ and a practice of minimal touch was encouraged in neonatal care, ${ }^{14}$ depriving infants of somatosensory stimulation that is now thought to be essential to neurodevelopment. ${ }^{6,30}$ Furthermore, early skin contact and positive affective tactile interventions such as kangaroo care ${ }^{13}$ and massage ${ }^{2,22}$ are thought to promote physiological stability, ${ }^{4,32,39}$ reduce clinical pain scores, $8,23,25$ and mitigate the attenuation of touch-evoked brain activity that has been associated with early-life pain. ${ }^{30}$ It is also important to note that pain experience is not dichotomous, and a limitation of this study is that pain discrimination was only considered at a single intensity with a relatively low saliency. It is plausible that if we were to consider a more intense noxious clinical procedure, such as chest drain insertion, discriminative facial expressions may be observed at an earlier gestation. Nevertheless, the demonstration of a lack of discriminative facial expressions after noxious and innocuous events in infants younger than 33 weeks' gestation highlights the need for caution when interpreting pain scores in the youngest infants. In this study, the noxious heel lance evoked a range of behavioural, neurophysiological, and autonomic responses, which were characterised across different durations lasting from milliseconds to seconds. The variation in the duration of these responses may, in part, explain why responses recorded using different measurement techniques may not necessarily be highly predictive of one another. ${ }^{34,37,43}$ Although a few of the younger gestation infants did display discriminative facial responses to the noxious procedure, these responses were less likely to occur than in infants approaching term gestation, and likely relate to the maturity of the individual's brain activity.

Consistent with previous research, ${ }^{12,19}$ we observed a developmental switch between immature delta brush responses and mature modality-specific evoked brain activity. This may be related to the disappearance of the subplate zone and the development of direct thalamocortical connections, as well as the formation of callosal and association pathways, which all occur during this developmental window. ${ }^{26}$ The emergence of discriminative facial expressions coincided with this developmental switch in brain activity. Further work will elucidate how the maturity of these structural pathways relates to sensory-evoked responses and the emergence of facial expression discrimination. The results of the study are limited by our choice of pain measures. Although the PIPP-R score is one of the best validated behavioural pain scores, using another behavioural measure would perhaps produce slightly different developmental timings for the emergence of discrimination. Furthermore, new measures of noxious-evoked brain activity are being developed, and time-frequency analysis of the EEG responses as well as imaging techniques, such as $\mathrm{FMRl}$, could be used to further explore changes in brain activity and localise the areas from which these developmental changes in activity originate.

It is interesting to consider the results of this study in the context of an evolutionary and practical ethics perspective. Evolutionarily, it may be considered unsurprising that premature and 


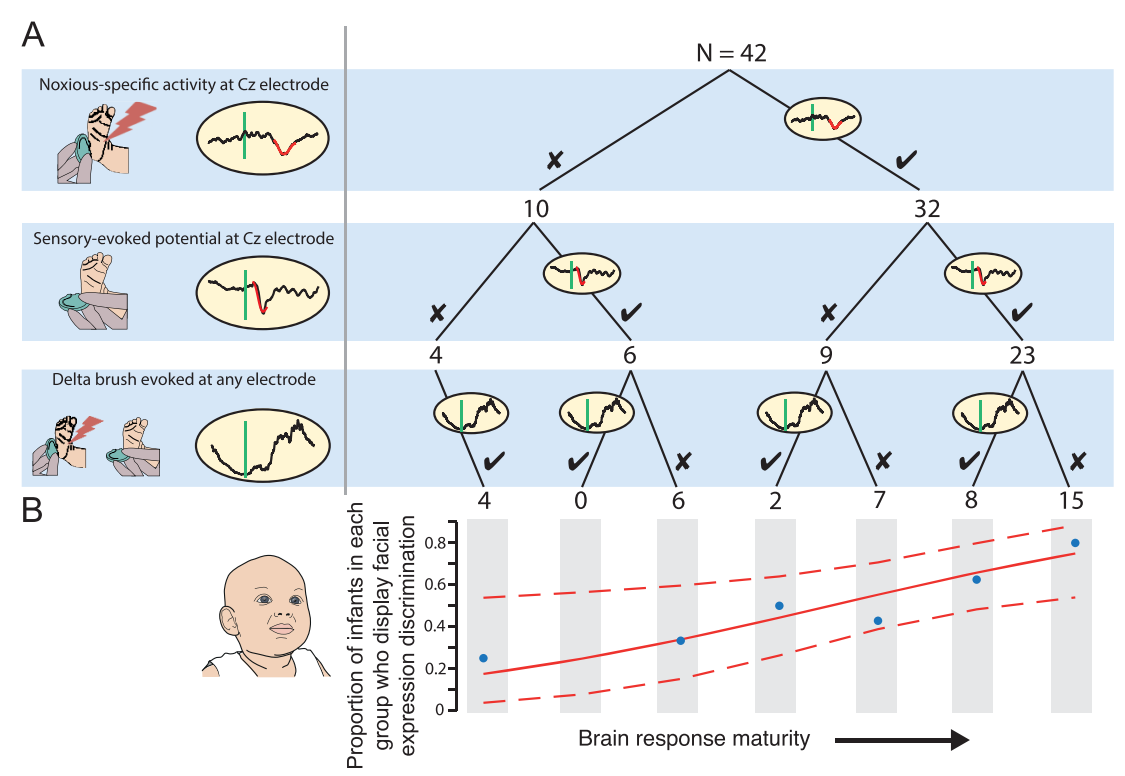

Figure 2. Facial expression discrimination depends on brain response maturity. (A) A decision tree was used to classify infants according to brain response maturity based on their responses to both noxious and innocuous stimuli. Noxious-specific brain activity and sensory-evoked potentials are seen in more mature, older infants. By contrast, delta brush responses are more immature. Therefore, infants classified on the right of the tree have more mature responses compared with infants on the left. The first split of the tree classifies infants dependent on the presence of noxious-specific brain activity; the second split classifies infants dependent on the presence of a sensory-evoked potential; and the final split considers whether delta brush activity was recorded at any electrode. Crosses indicate that a particular type of brain activity was not present, whereas ticks indicate that this type of brain activity was present. The number of infants classified at each split is indicated at the bottom of the branches. (B) Brain response maturity classification determined from the decision tree is plotted against the proportion of infants in each group that display facial expression discrimination (a response to the noxious stimulus but not the innocuous stimulus). The fit from a generalised linear model (solid lines) and 90\% confidence intervals (dashed lines) are overlaid.

neurologically immature infants are less likely to display discriminative behaviours. Facial expressions in infants are signals that evolve to elicit a response from caregivers. ${ }^{33}$ Essentially, as the evolution of these behavioural signals require a receiver, ${ }^{31}$ there can be no direct adaptive benefit for foetuses to use facial features to signal their experience of a noxious stimulus in utero. Thus, it is plausible that infants born very prematurely may also be less reliably able to display discriminative facial expressions after noxious and nonnoxious stimuli compared with more mature term-born infants. Facial expressions are complex behaviours, which have been observed to occur spontaneously and reflexively in utero from around 24 weeks' gestation and become increasingly complex with increasing gestation. ${ }^{36}$ Healthy foetuses, not subjected to aversive stimulation, show spontaneous facial expressions consistent with pain/distress, which are thought to be a sign of healthy maturation. ${ }^{36}$ It is possible, that these complex behaviours are essentially being practiced in utero, like other fundamental skills, such as breathing, as part of an adaptive developmental process that confers postnatal benefit to the foetus. ${ }^{20}$ The impact of ex utero life on the development of these behaviours is yet to be elucidated.

From an ethical perspective, a central priority for the care of preterm infants is the avoidance of unnecessary harm during treatment. Where it is uncertain whether harm may result, it is advisable to apply a precautionary principle that errs on the side of caution to prevent potential harms, even if scientific uncertainty exists about their extent. ${ }^{5,40}$ Our results raise fundamental questions as to how clinicians should best avoid potential harms to infants, many of which could arise from an incomplete understanding of pain. For example, failure to give infants analgesics in situations where they experience conscious pain could result in harm, but so too could providing analgesics with potential side effects in situations when they are unnecessary.
Although our data do not address the question of when infants are first capable of perceiving pain, the study does support the view that the brain's ability to discriminate noxious and innocuous tactile stimulation develops concomitantly with the emergence of differential behavioural responses to such stimuli. One possible interpretation of these results is that infants who respond facially to both noxious and innocuous tactile stimulation have a similar aversive experience in both cases. This view would suggest that painmitigating approaches for premature infants might be appropriate in the context of a wide range of procedures (including many not usually considered painful in older infants). At the other extreme, an alternative interpretation is that infants who are not able to discriminate between noxious and innocuous stimulation do not consciously experience pain. This view would support a much more restrictive use of analgesics in very premature newborn infants. However, regardless of whether noxious stimulation is consciously painful (which this study does not address), there is increasing evidence that painful procedures in early life are instrumentally harmful in that they can alter pain sensitivity and cognition in later life. ${ }^{21,45}$ Consequently, there is a critical need to limit procedures considered painful in prematurely born infants and to better manage and treat pain to mitigate long-term effects, irrespective of whether these procedures are perceptually painful.

In conclusion, we demonstrate that the emergence of facial expressions that discriminate between noxious and innocuous stimulation is concomitant with the maturation of brain responses in premature infants. This suggests that premature infants with relatively immature nervous systems display nondiscriminative facial behaviours to equally salient noxious and nonnoxious inputs, presenting challenges for the interpretation of pain and analgesia in this unique patient group. The urgent need for improved methodology to assess pain in the youngest premature infants is clear. 


\section{Conflict of interest statement}

The authors have no conflict of interest to declare.

\section{Acknowledgements}

The authors thank Maylis Carbajal for assistance with video scoring and Dr Robert Heathcote, an evolutionary biologist specialising in signal evolution, for his help with data interpretation.

This work was funded by The Wellcome Trust, United Kingdom. R. Slater is a Wellcome Trust Senior Research Fellow. G. Green is funded by the Oxford NIHR Biomedical Research Centre. E. Duff is a University of Oxford Excellence Fellow in Paediatric Neuroscience, supported by the SSNAP "Support for the Sick Newborn and their Parents" Medical Research Fund. A. Shriver receives funding from the Centre for Effective Altruism's EA Grants program. D. Wilkinson is supported by a grant from the Wellcome Trust. F. Moultrie is funded by the Wellcome Trust and the Oxford NIHR Biomedical Research Centre.

Author Contributions: R. Slater and C. Hartley designed the study. G. Green, A. Hoskin, C. Hartley, and F. Moultrie collected the data. C. Hartley, R. Slater, E. Duff, G. Green, and A. Hoskin analysed the data. All authors contributed to data interpretation and writing the manuscript.

\section{Appendix A. Supplemental digital content}

Supplemental digital content associated with this article can be found online at http://links.Iww.com/PAIN/A686.

\section{Supplemental video content}

Video content associated with this article can be found online at http://links.Iww.com/PAIN/A687.

\section{Article history:}

Received 27 June 2018

Received in revised form 3 September 2018

Accepted 4 September 2018

Available online 12 November 2018

\section{References}

[1] Ahola Kohut S, Pillai Riddell R. Does the neonatal facial coding system differentiate between infants experiencing pain-related and non-painrelated distress? J Pain 2009;10:214-20.

[2] Alvarez MJ, Fernandez D, Gomez-Salgado J, Rodriguez-Gonzalez D, Roson M, Lapena S. The effects of massage therapy in hospitalized preterm neonates: a systematic review. Int J Nurs Stud 2017;69: 119-36.

[3] Arichi T, Whitehead K, Barone G, Pressler R, Padormo F, Edwards AD, Fabrizi L. Localization of spontaneous bursting neuronal activity in the preterm human brain with simultaneous EEG-fMRI. Elife 2017;6: e27814.

[4] Bergman NJ, Linley LL, Fawcus SR. Randomized controlled trial of skinto-skin contact from birth versus conventional incubator for physiological stabilization in 1200- to 2199-gram newborns. Acta Paediatr 2004;93: 779-85.

[5] Birch J. Animal sentience and the precautionary principle. Anim Sentience: An Interdiscip J Anim Feeling 2017;2.

[6] Brauer J, Xiao YQ, Poulain T, Friederici AD, Schirmer A. Frequency of maternal touch predicts resting activity and connectivity of the developing social brain. Cereb Cortex 2016;26:3544-52.

[7] Carbajal R, Rousset A, Danan C, Coquery S, Nolent P, Ducrocq S, Saizou C, Lapillonne A, Granier M, Durand P, Lenclen R, Coursol A, Hubert P, de Saint Blanquat L, Boelle PY, Annequin D, Cimerman P, Anand KJ, Breart G. Epidemiology and treatment of painful procedures in neonates in intensive care units. JAMA 2008;300:60-70.
[8] Castral TC, Warnock F, Leite AM, Haas VJ, Scochi CG. The effects of skin-to-skin contact during acute pain in preterm newborns. Eur J Pain 2008;12:464-71.

[9] Chipaux M, Colonnese MT, Mauguen A, Fellous L, Mokhtari M, Lezcano O, Milh M, Dulac O, Chiron C, Khazipov R, Kaminska A. Auditory stimuli mimicking ambient sounds drive temporal "Delta-Brushes" in premature infants. PLoS One 2013;8:e79028.

[10] Colonnese MT, Kaminska A, Minlebaev M, Milh M, Bloem B, Lescure S, Moriette G, Chiron C, Ben-Ari Y, Khazipov R. A conserved switch in sensory processing prepares developing neocortex for vision. Neuron 2010;67:480-98.

[11] Cornelissen L, Fabrizi L, Patten D, Worley A, Meek J, Boyd S, Slater R, Fitzgerald M. Postnatal temporal, spatial and modality tuning of nociceptive cutaneous flexion reflexes in human infants. PLoS One 2013;8:e76470.

[12] Fabrizi L, Slater R, Worley A, Meek J, Boyd S, Olhede S, Fitzgerald M. A shift in sensory processing that enables the developing human brain to discriminate touch from pain. Curr Biol 2011;21:1552-8.

[13] Feldman R, Eidelman Al, Sirota L, Weller A. Comparison of skin-to-skin (kangaroo) and traditional care: parenting outcomes and preterm infant development. Pediatrics 2002;110:16-26.

[14] Field T, Hernandez-Reif M, Feijo L, Freedman J. Prenatal, perinatal and neonatal stimulation: a survey of neonatal nurseries. Infant Behav Dev 2006;29:24-31.

[15] Gibbins S, Stevens B, Beyene J, Chan PC, Bagg M, Asztalos E. Pain behaviours in extremely low gestational age infants. Early Hum Dev 2008; 84:451-8.

[16] Goldberg S. Social competence in infancy-model of parent-infant interaction. Merrill Palmer Quart 1977;23:163-77.

[17] Hartley C, Berthouze L, Mathieson SR, Boylan GB, Rennie JM, Marlow N, Farmer SF. Long-range temporal correlations in the EEG bursts of human preterm babies. PLoS One 2012;7:e31543.

[18] Hartley C, Duff EP, Green G, Mellado GS, Worley A, Rogers R, Slater R. Nociceptive brain activity as a measure of analgesic efficacy in infants. Sci Transl Med 2017;9:eaah6122.

[19] Hartley C, Moultrie F, Gursul D, Hoskin A, Adams E, Rogers R, Slater R. Changing balance of spinal cord excitability and nociceptive brain activity in early human development. Curr Biol 2016;26:1998-2002.

[20] Hepper P. Behavior during the prenatal period: adaptive for development and survival. Child Dev Perspect 2015;9:38-43.

[21] Hermann C, Hohmeister J, Demirakca S, Zohsel K, Flor H. Long-term alteration of pain sensitivity in school-aged children with early pain experiences. PAIN 2006;125:278-85.

[22] Hernandez-Reif M, Diego M, Field T. Preterm infants show reduced stress behaviors and activity after 5 days of massage therapy. Infant Behav Dev 2007;30:557-61.

[23] Jain S, Kumar P, McMillan DD. Prior leg massage decreases pain responses to heel stick in preterm babies. J Paediatr Child Health 2006; 42:505-8.

[24] Johnston CC, Stevens BJ, Yang F, Horton L. Differential response to pain by very premature neonates. PAIN 1995;61:471-9.

[25] Kashaninia Z, Sajedi F, Rahgozar M, Noghabi FA. The effect of Kangaroo care on behavioral responses to pain of an intramuscular injection in neonates. J Spec Pediatr Nurs 2008;13:275-80.

[26] Kostovic I, Jovanov-Milosevic N. The development of cerebral connections during the first 20-45 weeks' gestation. Semin Fetal Neonat M 2006;11:415-22.

[27] Kunz M, Mylius V, Schepelmann K, Lautenbacher S. On the relationship between self-report and facial expression of pain. J Pain 2004;5:368-76.

[28] Lim Y, Godambe S. Prevention and management of procedural pain in the neonate: an update, American Academy of Pediatrics, 2016. Arch Dis Child Educ Pract Ed 2017;102:254-6.

[29] Long JG, Philip AGS, Lucey JF. Excessive handling as a cause of hypoxemia. Pediatrics 1980;65:203-7.

[30] Maitre NL, Key AP, Chorna OD, Slaughter JC, Matusz PJ, Wallace MT, Murray MM. The dual nature of early-life experience on somatosensory processing in the human infant brain. Curr Biol 2017;27:1048-54.

[31] Maynard-Smith J, Harper D. Animal signals. Oxford, United Kingdom: Oxford University Press, 2003.

[32] Mitchell AJ, Yates C, Williams K, Hall RW. Effects of daily kangaroo care on cardiorespiratory parameters in preterm infants. J Neonatal Perinatal Med 2013;6:243-9.

[33] Pillai Riddell R, Racine N. Assessing pain in infancy: the caregiver context. Pain Res Manag 2009;14:27-32.

[34] Ranger M, Johnston CC, Anand KJ. Current controversies regarding pain assessment in neonates. Semin Perinatol 2007;31:283-8.

[35] Rasmussen CE, Williams CKI. Gaussian process for machine learning. MIT press, 2006. 
[36] Reissland N, Francis B, Mason J, Lincoln K. Do facial expressions develop before birth? PLoS One 2011;6:e24081.

[37] Slater R, Cantarella A, Franck L, Meek J, Fitzgerald M. How well do clinical pain assessment tools reflect pain in infants? PLoS Med 2008;5:e129.

[38] Slater R, Worley A, Fabrizi L, Roberts S, Meek J, Boyd S, Fitzgerald M. Evoked potentials generated by noxious stimulation in the human infant brain. Eur J Pain 2010;14:321-6.

[39] Smith SL, Lux R, Haley S, Slater H, Beachy J, Moyer-Mileur LJ. The effect of massage on heart rate variability in preterm infants. J Perinatol 2013; 33:59-64.

[40] Steel D. The precautionary principle and the dilemma objection. Ethics Policy Environ 2013;16:321-40.

[41] Stevens B, Johnston C, Petryshen P, Taddio A. Premature infant pain profile: development and initial validation. Clin J Pain 1996;12:13-22.
[42] Stevens BJ, Gibbins S, Yamada J, Dionne K, Lee G, Johnston C, Taddio A. The premature infant pain profile-revised (PIPP-r) initial validation and feasibility. Clin J Pain 2014;30:238-43.

[43] Verriotis M, Fabrizi L, Lee A, Cooper RJ, Fitzgerald M, Meek J. Mapping cortical responses to somatosensory stimuli in human infants with simultaneous near-infrared spectroscopy and event-related potential recording. eNeuro 2016;3:ENEURO.0026-16.2016.

[44] Verriotis M, Jones L, Whitehead K, Laudiano-Dray P, Panayotidis I, Patel H, Meek J, Fabrizi L, Fitzgerald M. The distribution of pain activity across the human neonatal brain is sex dependent. Neurolmage 2018;178:69-77.

[45] Vinall J, Grunau RE. Impact of repeated procedural pain-related stress in infants born very preterm. Pediatr Res 2014;75:584-7.

[46] Worley A, Fabrizi L, Boyd S, Slater R. Multi-modal pain measurements in infants. J Neurosci Meth 2012;205:252-7. 